\title{
A counter-example to the theorem of Hiemer and Snurnikov
}

\author{
Thierry Monteil *
}

\begin{abstract}
A planar polygonal billiard $\mathcal{P}$ is said to have the finite blocking property if for every pair $(O, A)$ of points in $\mathcal{P}$ there exists a finite number of "blocking" points $B_{1}, \ldots, B_{n}$ such that every billiard trajectory from $O$ to $A$ meets one of the $B_{i}$ 's. As a counter-example to a theorem of Hiemer and Snurnikov, we construct a family of rational billiards that lack the finite blocking property.
\end{abstract}

Key words: rational polygonal billiards, translation surfaces, blocking property.

\section{Introduction}

A planar polygonal billiard $\mathcal{P}$ is said to have the finite blocking property if for every pair $(O, A)$ of points in $\mathcal{P}$ there exists a finite number of "blocking" points $B_{1}, \ldots, B_{n}$ (different from $O$ and $A$ ) such that every billiard trajectory from $O$ to $A$ meets one of the $B_{i}$ 's.

In [HS], Hiemer and Snurnikov tried to prove that any rational polygonal billiard has the finite blocking property. The aim of this paper is to construct a family of rational billiards that lack the finite blocking property.

\footnotetext{
${ }^{*}$ Institut de Mathématiques de Luminy, CNRS UPR 9016, Case 907, 163 Avenue de Luminy, 13288 Marseille cedex 09, France - E-Mail: monteil@iml.univ-mrs.fr - Tel: +33491269677- Fax : +334 91269655
} 


\section{The counter-example}

Let $\alpha$ be a positive irrational number and $\mathcal{P}_{\alpha}$ be the polygon drawn in Figure $1\left(L_{1}\right.$ and $L_{2}$ can be chosen arbitrarily, greater than 1 ).

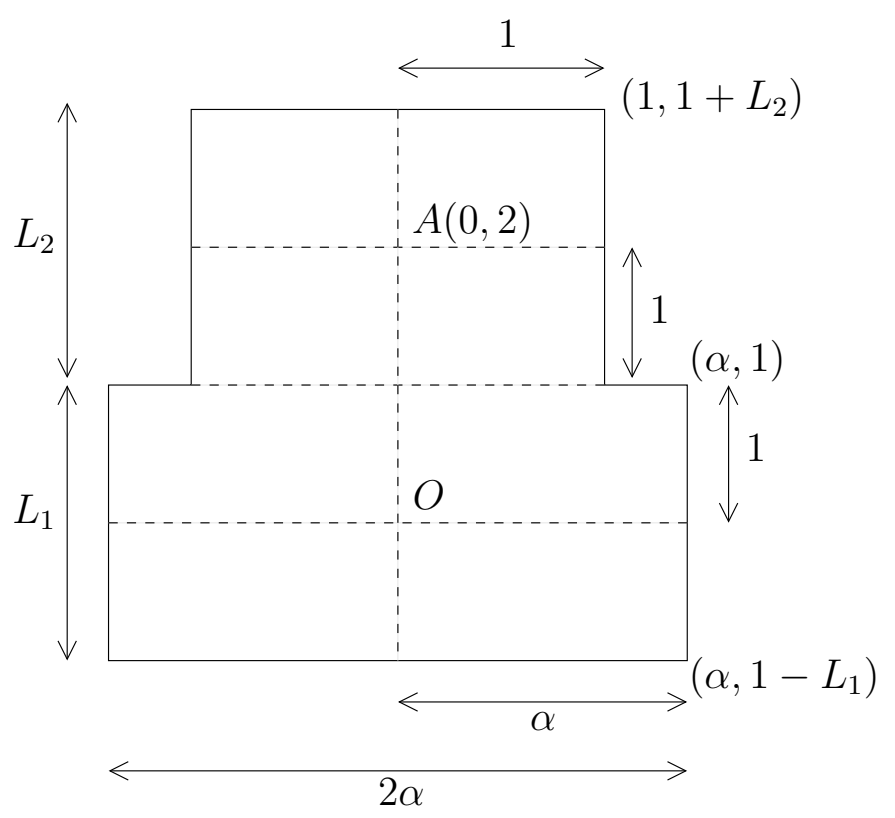

Figure 1: The polygon $\mathcal{P}_{\alpha}$.

Let $\left(p_{n}, q_{n}\right)_{n \in \mathbb{N}}$ be a sequence in $\mathbb{N}^{* 2}$ such that:

- $q_{n}$ is strictly increasing

- $\left|p_{n}-q_{n} \alpha\right|<1$

For example, we can take $q_{n}=n+1$ and $p_{n}=\left[q_{n} \alpha\right]$.

For $n \in \mathbb{N}$, let $\gamma_{n}$ be the billiard trajectory starting from $O$ to $A$ with slope

$$
\frac{1}{p_{n}+q_{n} \alpha}=\frac{1}{2 q_{n} \alpha+\lambda_{n}}=\frac{1}{2 p_{n}-\lambda_{n}}
$$

where $\left.\lambda_{n}=p_{n}-q_{n} \alpha \in\right]-1,1[$.

So, we can check (with the classical unfolding procedure shown in Figure 2) that $\gamma_{n}$ hits $q_{n}$ walls, passes through $\left(\lambda_{n}, 1\right)$, hits $p_{n}$ walls and then passes through $A(0,2)$. 


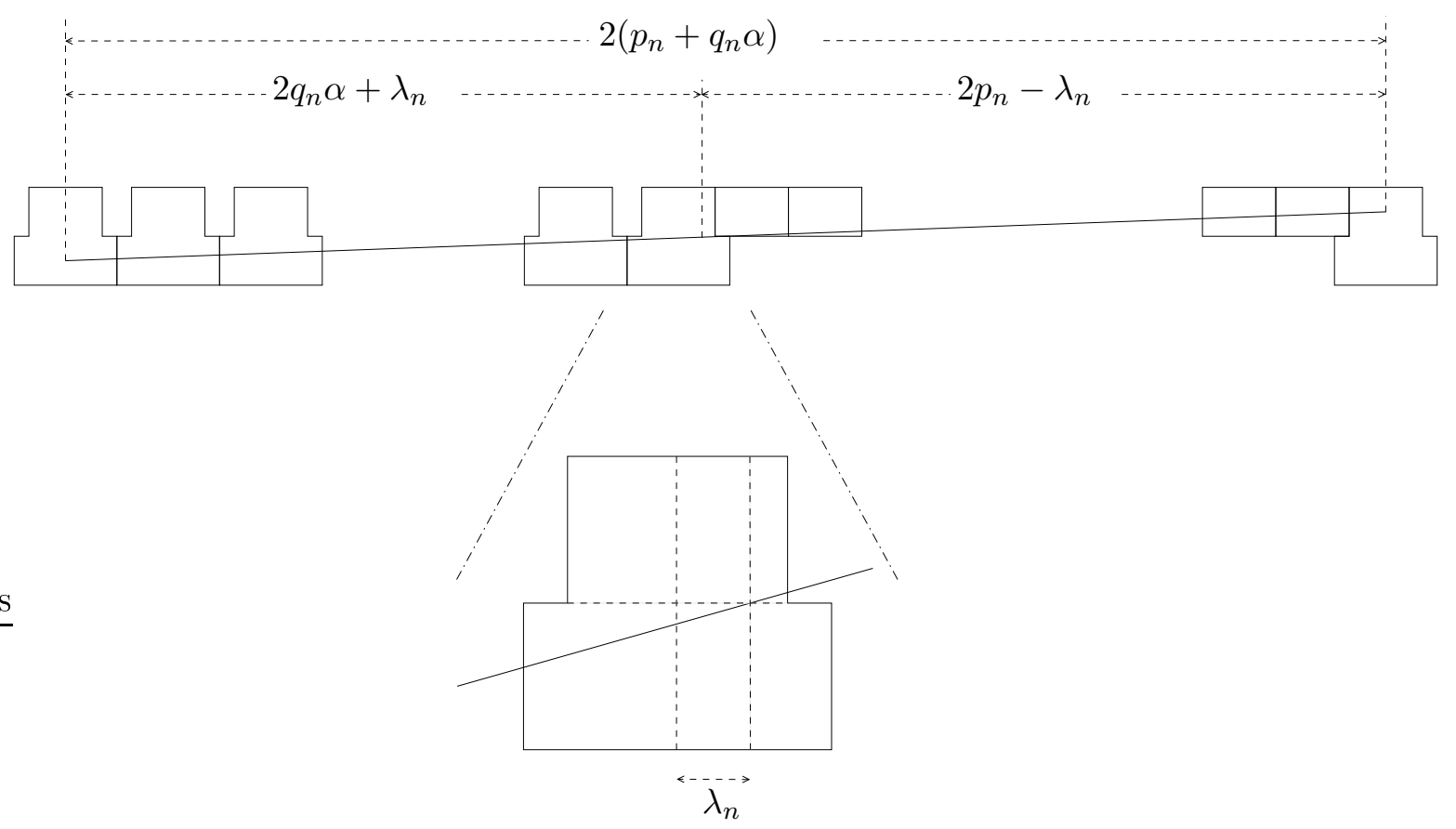

Figure 2: The unfolding procedure.

The fact that $\left.\lambda_{n} \in\right]-1,1[$ enables us to avoid the banana peel shown in Figure 3 ,

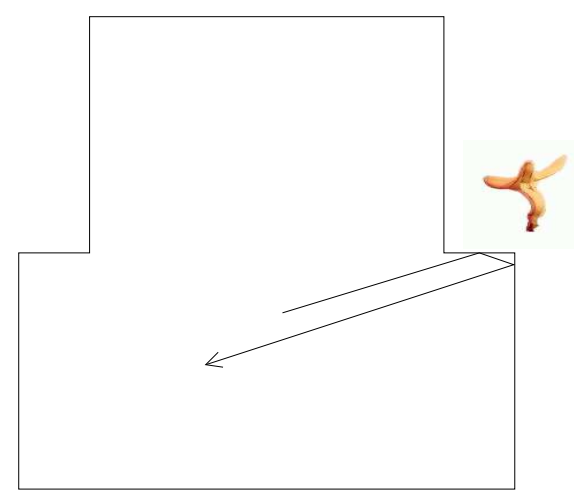

Figure 3: The banana peel.

Now, we assume by contradiction that there is a point $B(x, y)$ in $\mathcal{P}_{\alpha}$ distinct from $O$ and $A$ such that infinitely many $\gamma_{n}$ pass through $B$. Hence, there is a subsequence such that for all $n$ in $\mathbb{N}, \gamma_{i_{n}}$ passes through $B$.

There are two cases to consider:

First case: $y \in] 0,1]$. By looking at the unfolded version of the trajectory (Figure 21), we see that $x=\varepsilon_{i_{n}} y\left(p_{i_{n}}+q_{i_{n}} \alpha\right)[\bmod 2 \alpha]$ where $\varepsilon_{i_{n}} \in\{-1,1\}$ depends on the parity of the number of bounces of $\gamma_{i_{n}}$ from $O$ to $B$.

So, there exists a sequence $\left(k_{n}\right)_{n \in \mathbb{N}}$ in $\mathbb{Z}$ such that $x=\varepsilon_{i_{n}} y\left(p_{i_{n}}+q_{i_{n}} \alpha\right)+2 k_{i_{n}} \alpha$. 
Taking a further subsequence, we can consider $\varepsilon \circ i$ to be constant with value $\varepsilon$.

We have $x=\varepsilon y\left(p_{i_{0}}+q_{i_{0}} \alpha\right)+2 k_{i_{0}} \alpha=\varepsilon y\left(p_{i_{1}}+q_{i_{1}} \alpha\right)+2 k_{i_{1}} \alpha$.

Hence, $\left(p_{i_{1}}-p_{i_{0}}\right)+\left(q_{i_{1}}-q_{i_{0}}\right) \alpha=\frac{\varepsilon 2 \alpha}{y}\left(k_{i_{0}}-k_{i_{1}}\right) \neq 0$.

So, $\frac{\varepsilon 2 \alpha}{y}$ can be written as $r+s \alpha$ where $r$ and $s$ are rational numbers.

Now, if $n \geq 1$, we still have $\left(p_{i_{n}}-p_{i_{0}}\right)+\left(q_{i_{n}}-q_{i_{0}}\right) \alpha=(r+s \alpha)\left(k_{i_{0}}-k_{i_{n}}\right)$.

Because $(1, \alpha)$ is free over $\mathbb{Q}$, we have

- $\left(p_{i_{n}}-p_{i_{0}}\right)=r\left(k_{i_{0}}-k_{i_{n}}\right)$

- $\left(q_{i_{n}}-q_{i_{0}}\right)=s\left(k_{i_{0}}-k_{i_{n}}\right) \neq 0$ (remember that $q_{n}$ is strictly increasing)

Thus, by dividing,

$$
\frac{r}{s}=\frac{p_{i_{n}}-p_{i_{0}}}{q_{i_{n}}-q_{i_{0}}}=\frac{p_{i_{n}}}{q_{i_{n}}}\left(1-\frac{p_{i_{0}}}{p_{i_{n}}}\right)\left(\frac{1}{1-\frac{q_{i_{0}}}{q_{i_{n}}}}\right) \underset{n \rightarrow \infty}{\longrightarrow} \alpha \in \mathbb{R} \backslash \mathbb{Q}
$$

leading to a contradiction.

For the second case, if $y \in[1,2[$, it is exactly the same (take the point $A(0,2)$ as the origin and reverse Figure 2).

Thus, the billiard $\mathcal{P}_{\alpha}$ lacks the finite blocking property. 


\section{Conclusion}

In [M], we study Hiemer and Snurnikov's proof: it works for rational billiards with discrete translation group (such billiards are called almost integrable). Then we generalize the notion of finite blocking property to translation surfaces (see [MT] for precise definitions). With an analogous construction to the one described above, we obtain the following results:

Theorem 1 Let $n \geq 3$ be an integer. The following assertions are equivalent:

- the regular n-gon has the finite blocking property.

- the right-angled triangle with an angle equal to $\pi / n$ has the finite blocking property.

- $n \in\{3,4,6\}$.

Theorem 2 A translation surface that admits cylinder decomposition of commensurable moduli in two transversal directions has the finite blocking property if and only if it is a torus branched covering.

Corollary 1 A Veech surface has the finite blocking property if and only if it is a torus branched covering.

Note that torus branched coverings are the analogue (in the vocabulary of translation surfaces) of almost integrable billiards.

We also provide a local sufficient condition for a translation surface to fail the finite blocking property: it enables us to give a complete classification for the L-shaped surfaces and a density result in the space of translation surfaces in every genus $g \geq 2$.

\section{References}

[HS] P. Hiemer, V. Snurnikov, Polygonal billiards with small obstacles, Journal of Statistical Physics, Vol 90 (1998), p 453-466.

[MT] H. Masur, S. TABACHNIKOV, Rational billiards and flat structures, Handbook on dynamical systems, Vol. 1A, p 1015-1089, North-Holland, Amsterdam, (2002).

[M] T. MonteIL, On the finite blocking property, preprint. 